\title{
Zur Lehre vom Roseschen Kopftetanus.
}

Von Dr. Julius Friedländer, Nervenarzt, und

Dr. E. v. Meyer, Chirurg am Diakonissenhaus in Frankfurt a.M.

Die interessante und relativ seltene Abart des Wundstarrkrampfes, die nach dem Namen ihres Entdeckers als Rosescher Kopftetanus bezeichnet wird, ist in der wissenschaftlichen Literatur der letzten Jahrzehnte recht häufig beschrieben worden, um so mehr, als sich Vertreter von vier Gebieten der Medizin, Chirurgen, Interne, Bakteriologen und Neurologen mit ihrem Studium befassen. Indessen zeigt die Physiognomie dieser Krankheitsform, wenn auch in ibren Hauptzïgen feststehend, doch im einzelnen so mannigfaltige Varietäten und im ganzen so vieles noch Unaufgeklärte, daß die Mitteilung eines besonders charakteristischen und sorgfältig beobachteten Falles wohl gerechtfertigt orscheinen darf.

Die Krankengeschichte ist, kurz zusammengefaßt und auf das wesentliche beschränkt, folgende: Der sehr kräftige, zuvor stets gesunde, 23 jährige Fuhrmann P. U. aus Griesheim a. M. verunglückte ain 7. November v. J., indem er infolge Scheuwerdens der Pferde von seinem Wagen herabgeschleudert und eine Strecke weit geschleift wurde. Er stürzte vornüber auf die sehr schmutzige Landstraße und zog sich beim Auffallen auf das Gesicht mehrere Verletzungen in der Umgebung des rechten Auges zu, wegen deren er sich baldigst in Behandlung von Dr. Stieb in G. begab. Am 13. November abends wurde er von diesem mit der Diagnose: Trismus in das Frankfurter Diakonissenhaus eingewiesen und kam dort in die Behandlung von Dr. v. Meyer, der Dr. Friedländer zur neurologischen Beobachtung zuzog. Irgendwelche Innervationsstörungen im Gesicht, insbesondere Zuckungen, Spasmen oder Kontrakturen hatten bis dahin in der Muskulatur beider Gesichtshälften nicht bestanden. Bei der Aufnahme wurde am rechten Arcus superciliaris längs der Augenbraue eine $3 \mathrm{~cm}$ lange, $1 / 2 \mathrm{~cm}$ breite, bis auf das Periost gehende, gequetschte Rißwunde konstatiert und in Höhe des rechten unteren Orbitalrandes am unteren Lid eine daumennagelgroße, oberflächliche Hautabschürfung - beide mit schmierigem, fibrinösem Belag bedeckt. Diese zweifellos infizierten Wunden wurden sofort mit Chlumskischem Karbolkampfer energisch desinfiziert. Im übrigen bestand keine entzündliche Reaktion, kein Fieber und am ganzen behaarten und unbehaarten Schädel keine Schmerzhaftigkeit, Schwellung oder Druckempfindlichkeit, die auf eine äußere oder innere Verletzung hingewiesen hätte, nur zeigten sich beiderseits die Kaumuskeln bretthart kontrahiert, und wenn auch noch keine vollständige Kieferklemmo vorlag, so war doch durch häufig eintretende, sehr schmerzhaft empfundene, klonische Kinnbackenkrampfe das Sprechen und Kauen schon fast unmöglich. Am nächsten Morgen fällt vor allem eine ausgeprägte rechtseitige Facialisluhmung auf: die ganze rechte Gesichtshalfte ist, während die linke mimisch korrekt funktioniert und keinerlei Spannung oder Kontraktur zeigt, völlig schlaff und unbeweglich, soweit ihre Muskulatur von Stirn-, Wangen- und Mundästen des Facialis innerviert wird. Die rechte Nasolabialfalte ist gänzlich verstrichen, die Nasenspitze steht etwas nach links, der rechte Mundwinkel hängt herab, der Mund ist stark nach der gesunden Seite verzogen und kann nicht zum Pfeifen gespitzt werden; die Nase kann rechterseits nicht gerumpft, die Stirn rechts nicht gerunzelt, die rechte Augenbraue nicht gehoben, das rechte $\mathrm{Ohr}$ nicht bewegt werden. Die Sensibilităt der Haut ist im Bereich der Lähmung intakt, doch lösen Nadelstiche keine Reflexbewegung in den gelahmten Muskeln aus; das Gehör ist nicht alteriert; eine Geschmacksprüfung war wegen der vorhandenen Kieferklemme unausfïhrbar; ebenso mußte eine elektrische Prüfung unterbleiben, da sie vermơge der stark erhöhten Reflexerregbarkeit sofort die schmerzhaften Trismusanfäle provoziert hätte. Auch von einer näheren Untersuchung der äußeren Augenmuskeln mußte mit Rücksicht auf das kollaterale Oedem und die benachbarten, noch offenen Wunden vorerst Abstand genommen werden: nur der wichtige Befund wurde sicher festgestellt, daß das rechte obere Lid schlaff herabhing und so das rechte Auge ständig ganz geschlossen war, somit die zum M. orbicularis palpebrar. gehenden Zweige des N. facialis nicht mitgelähmt waren. Dio Beweg. lichkeit des Nackens zeigt sich in keiner Weise eingeschränkt: der Patient kann das Kinn auf die Brust herabsenken; ebenso sind die Bewegungen des Kopfes nach hinten und beiden Seiten aktiv und passiv leicht auszufuhren. Auch fehlt jede Versteifung in den Bauchmuskeln; die vier Fxtremitaten sind vollkommen frei beweglich. Das Sensorium ist nicht getrübt.

Dio Behandlung bestand bis zum Eintreffen des in Höchst bestellten Behringschen Tetanusserums in der Anwendung von Chloralklystieren zu je $2 \mathrm{~g}$ und centigrammweisen Morphiuminjektionen. Nacl Empfang des Antitoxins wurden am 15. November 
abends und am 16 vormittags je 100 Antitoxineinheiten unter antiseptischen Kautelen in die Nackenhaut injiziert. Da sich indessen innerhalb der nachsten 24 Stundeu keinerlei örtliche oder allgemeine Reaktion daranf zeigte. aulser daß die zuvor normale Temperatur auf 39.1 anstieg. wurde von weiterer Antitoxinbehandlung abgesehen und die Chloral-Morphinmtherapie defınitiv wieder aufgenommen. Inzwischen hatten sich allmählich zunehmende Schlingbeschwerden eingestellt. sodal3 selbst das Schlucken von Flüssigkeiten. wie Milch und Suppe, nicht immer gelang, $11 \mathrm{~m}$ so mehr, als die Mundsperre einen solchen Grad erreicht hatte. daß kaum ein kleiner Löffel zwischen die Zähne gedrängt werden konnte: nicht selten kam es auch vor, daß ein Finger. den der Patient zufällig nach dem Munde geführt hatte, einen heftigen Krampf der Kinnbacken ausloste und sich dabei zwischen den Zahnreihen so festklemmte. daß er nur mit Gewalt wieder entfernt werden konnte. Am 18. November waren die Wunden in der Umgebung des rechten Anges, die die Eingangspforte des Tetanusgiftes gebildet hatten, vollständig geheilt. sodaß3 nun eine genauere Untersuchung des Auges und seiner Muskulatur vorgenommen werden kann. Der Befund stellt sich folgendermalien dar: Während das linke Auge normal geöffnet und geschlossen wird. der Bulbus nach allen Seiten frei beweglich ist und eine durch die starken Narkotica verengte Pupille zeigt, besteht rechterseits eine totale Ptosis: das rechte obere Liul hängt infolge Lähmung des M. levator palpebr. sup. schlaff und unbeweglich bis über den unteren Cornealrand herab und fällt auch bei passiver Hebung sofort wieder hernieder: die Pupille ist infolge Lähmung des Sphıncter iridis erweitert und reaktionslos. der Bulbus ist starr geradeaus fixiert und nach oben, unten, innen und außen unbeweglich. Es besteht somit rechterseits eine komplette Oculomotorins- und Abducenslathmung. vielleicht auch Trochlearjslähmnng. Im weiteren Verlanfe der Krankheit steigert sich besonders die stơrung des Schlingaktes derart. daß jeder Versuch. ja jede Intention zu einer Schluckbewegung vermöge der enorm erhöhten Reflexerregbarkeit des Rachens die heftigsten Schlingkrämpfe auslóst. Diese außerordentlich schmerzhaften Spasmen des Oesophagns sind oft mit Inspirationskrämplen verbunden: der Patient baumt sich dabei förmlich im Bett auf, sein 'Thorax bleibt sekundenlang in äußerster Inspirationsstellung stehen. sein Gesicht wird cyanotisch und bedeckt sich mit Schweiß. Aber selbst auf diesem Höhepunkte der Erkrankung fehlt merkwürdigerweise jede Beteilignng der Nackenmuskulatur, die nach wie vor keine Spur von Starre. Spannung oder Schmerzhaftigkeit aufweist. Dagegen macht sich in diesen 'T'agen eine sehr lastige Salivation geltend, indem ein ziemlich dicker. mit Schleim vermengter Speichel aus dem gelăhmten rechten Mundwinkel beständig in Menge ausflieBt. Da unter solchen Umständen dem Kranken per os nichts mehr beizubringen war und Nährklystiere angewendet werden mußten, traten allmählich unter leichten Fieberregungen und Beschleunigung von Puls und Atmung Inanitionserscheinungen ein und. ohne daß sich der Tetanus auf Nacken-. Rumpf- und Extremitätenmuskeln fortgesetzt hätte. folgte nun etwa am 21. November unter Sistierung des Spejchelflusses dasjenige Stadium der Krankheit, das sich mit Lösung des 'l'rismns und schlaffem Herabsinken des Unterkiefers eiuleitet und als terminales Erschöpfungsstadium von Rose charakterisiert worden ist. Bei fortbestehender Facialislähmung beschloß am 25. November unter steigendem Kräfteverfall. Inkontinenz von Blase und Mastdarm. aber bei ungetrübtem Sensorium der Exitus letalis die. einschließlich der siebentägigen Inkubationszeit. 19 tägige Krankheit. Die Sektion konnte nicht gemacht werden.

Was zunächst die Diagnos e betrifft. so kann keine Frage sein, daß hier in typischer Form das Krankheitsbild vorliegt. das zuerst Rose (1) als ..Kopf-" (oder „Facialis-") Tetanus mit der Erläuterung aufstellte, daß es eine von Kopfwunden. meist in der Orbitalgegend ausgehende Sonderart des Tetanus sei, bei der außer den in gewöhnlicher Weise mit Trismus beginncnden und weiterhin die Nacken- und Rumpimuskulatur ergleifenden. tonischen Krämpfen eine Lähmung des Facialis auf der Seite der Verletzung auftritt. In diese Kategorie des Wundstarrkrampfes gehört. trotz des Fehlens der Nackenstarre, nach Aetiologie, klinischem Bild und Verlauf zweifellos der vorliegende Fall. und wenn man ihn im Sinne Roses noch näher rubrizieren wollte, so wülde er wegen der vorherrschenden Schlingkrämpfo in die besondere Klasse des Kopftetanus einzureihen sein. die Roso wegen ihrer Aehnlichkeit mit der Hydrophobie als "Tetanus hydrophobicoides" bezeichnet hat. (2)

Eröltern wir nun die Symptomatologie unseres Falles im einzelnen etwas näher, so steht im Vordergrunde des Interesses die Facialislähmung: Inmitten einer Szene, die von Krampferscheinungen schwerster Art beherrscht wird. ist das Auftreten dieser meist isolierten Lähmung ein so paradoxes und schwer erklärliches Phänomen. daf es von jeher die besondere Aufmerksamkeit aller Beobachter erregt und die Frage seiner Herkunft zu einem förmlichen Konflikt unter ihnen geführt hat. Welches ist denn nun der Sitz und Charakter dieser Facialislähmung. die wir beim Kopftetanus nicht konstant. aber doch so oft beobachten, daß eine zufällige Koinzidenz ausgeschlossen ist? Ist sie zentralen oder peripherischen. intrakraniellen oder extrakraniellen Ursprunges? Und welches ist ihre Aetiologie und Pathogenese? Ist es eine traumatische. eine reflektorische. eine toxische oder eine neuritische Lăhmung?

In welcher Partie seines Verlaufes der Facialis gelähmt ist. dürfte im vorliegenden Falle, obwohl auf eine elektrische Untersuchung des Nerven verzichtet werden mußte, nicht schwer zu entscheiden sein: Das Mitbefallensein des Stirnfacialis. das Fehlen aller Hirnsymptome. das Aufgehobensein aller Reflexe sprechen unzweifelhaft für eine peripherische Läsion. Diese läßt sich aber noch genauer lokalisieren: da einerseits jede Gehörsstörung fehlt. anderseits das rechte Ohr unbewegbar. somit der N. auricularis post. mitgelähmt ist. muß der Ort der Leitungsunterbrechung intrakraniell innerhalb des Canalis Fallopiae sein.

Welcher $\mathrm{Natur}$ ist nun die Lähmung? Daß sie unmittelbar durch den Sturz entstanden, eine direkt traumatische ist, darf von volnhelein ausgeschlossen werden: denn eine Facialislähmung, die nahezu das ganze Gebiet des Nerven einschlieflich seiner Mundäste umfaßt. könnte wohl direkt aus einem 'Trauma resultieren. das die Gegend der Parotis oder des Ohres getroffen hätte, aber aus dem Chok eines Falles auf die Orbita, der nachweislich auber zwei Wunden in der Nähe des Auges keinerlei Schädigungen hervorgerufen hat. kann eine Facialislähmung von dieser Ausdehnung unmöglich erklärt werden. Die Möglichkeit. daß die Lähmung allein dem mechanischen Insult des Traumas ihre Entstehung verdankt, muß somit auf Grund anatomischer Erwägungen von der Hand gewiesen werden.

Könnte die Lähmung nun eine reflektorische sein? Diese Ansicht ist vor allem von Nothnagel (3) gelegentlich der klinischen Vorstellung eines einschlägigen Falles im Jahre 1901 vertreten worden. Es dürften ihr jedoch schwerwiegende Bedenken gegeniiberstehen: wenn man überhaupt an die Existenz von reflektierten Funktionsstörungen. von rein funktionellen. jedes anatomischen Substrates entbehrenden Lähmungen durch Fernwirkung glauben will. so würde man sich den Vorgang in unserem und ähnlichen Fällen so vorzustellen haben, daß ein peripherischer sensibler Reiz sich zentripetal fortleitet und im Zentralorgan reflektorisch eine Lähnung auslöst. Es würde danu freilich kein echt paralytischer, primár aus krankhaften Veränderungen im motorischen Nervenapparat hervorgegangener Zustand vorliegen. sondern vielmehr eine Hemmung im Sinne einer zentral bedingten Aufhebung der motorischen Innervation. Eine solche reflektorische Hemmung oder Lahmung könnte aber erstens bei dem bilateral-symmetrisch innervierten System der mimischen Gesichtsmuskulatur nicht einseitis auftreten und zweitens nicht entgegen ihrer zentralen Herkunf die klinischen Merkmale einer peripherischen Lähmung, wie in unserm Falle. aufweisen. Fragen wir weiter, wo wäre hier der sensible Reiz. stark genug, um eine Facialisparalyse reflektorisch auszulösen - so könnte man, da der ganz geringfügige Wundschmerz als solcher nicht in Betracht kommen kann, nur annehmen, daA die heftige und anhaltende Irritation sensibler Trigeminusfasern, die den Trismus begleitet und so sehr schmerzhaft macht den Anstoß $\mathrm{zu}$ einer so schweren Reaktion auf motorischem Gebiete geben könnte. Aber auch diese Annahme ist unhaltbar, denn es sind in der Literatur des Kopftetanus (4) zahlreiche Fälle beschrieben. in denen die Facialislähmung schon vor dem Trismus bestanden hat. Die ganze Auffassung. daß die Facialislähmung hier reflektorischer Natur ist. muß somit aus den obigen Griinden verworfen werden.

Eine dritte Hypothese, deren Urheber Oliva (5) und deren hervorragendster Verteidiger Jolly (6) gewesen ist. hallt die Lähmung für eine toxische. "Das Tetanotoxin - so ist in aller Kürze ihre Argumentation - besitzt. wie bakteriologisch festgestellt ist, nicht nur eine besondere Affinität zum Zentralnervensystem. sondern auch eine Wanderungsfähigkeit in den peripherischen Nerven, die ihm als Angriffspunkt dienen, und kann. auf ihrer Bahn zentripetal bis zu den Nervenkernen fortwirkend (7) oder fortgeleitet (8), Lähmungen erzengen. So entstehe auch die Labmung beim Facialis, indem das Tetanusgift in seine ganz oberflächlich gelegenen 
Endausbreitungen, die bei Wundverletzungen an den Orbitalrändern bloßgelegt seien, direkt inokuliert werde. ${ }^{*}$ Auch gegen diese Ansicht wird manches einzuwenden sein: Dab das Tetanotoxin primar Krämpfe erzeugt, Trismus auf der Bahn motorischer V.-Aeste, Tetanus auf dem Weg motorischer Rumpf- und Extremitätennerven, ist als unumstößliche Tatsache experimentell festgestellt (9). Daß dasselbe Virus aber nur gerade bei e in em motorischen Nerven, dem Facialis, eine primäre Lähmung, das gleiche schädliche Agens also uberall Reizung und nur hier Lähming bewirken soll, ist widerspruchsvoll und schwer zu glauben. Zwar haben Brieger und Fränkel (10) im Laboratorium nachgewiesen, daß das Tetanotoxin sowohl Krämpfe wie Lähmungen hervorrufen kann, und Brunner (11) hat gezeigt, daßs bei Kaninchen und Meerschweinchen große Mengen des Giftes lokale Lähmungserscheinungen in den der lmpfstelle benachbarten Gesichtsmuskeln zur Folge haben. Aber man mag den Vergleichswert des Tierversuches hoch oder niedrig anschlagen, bier ist sein Ergebnis gewiß nicht beweiskräftig: Denn Brunner hebt selbst hervor, dab bei seinen Versuchstieren ein ausgesprochen lokales Phänomen vorliegt und die Lähmung sich nur auf unmittelbar der Impfstelle benachbarte Gesichtsmuskeln erstreckt. In Fällen wie der unsrige aber stellt die Lähmung gerade keine lokale Affektion dar: denn sie betrifft die von der Infektionsstelle entfernteren Stirn-, Wangen- und Mundäste des Facialis, und läßt die ihr am allernächsten gelegenen, oculopupillären Fasern frei. Der Widerspruch löst sich somit auf diese Weise nicht. Einen vermittelnden Standpunkt in der Frage nimmt u. a. Oppenheim (12) ein: er hält die Lähmung nicht für primär und meint, daß ihr stets ein Reizungszustand im Nerven vorangegangen sei. Indessen ist ein solches Vorstadium nur in einigen wenigen in der Literatur niedergelegten Fallen beobachtet worden, und speziell in unserem Falle hat der vorbehandelnde Arzt Dr. Stieb (13) das Fehlen jeder Spur von Spasmen oder Kontraktur vor der Lähmung besonders konstatiert. Die Annahme Oppenheims hat somit wenig Wahrscheinlichkeit für sich und vermag auch keinen befriedigenden Aufschluß zu geben. Ebenso ist damit, daß man eine nbesondere Affinität des N. facialis für die lähmenden Bestandteile des Tetanotoxins", wie Solmsen (14), oder "eine spezifisch geringere Widerstandsfahigkeit des N. facialis gegen das Tetanusgift", wie K l e m m (15), supponiert, durchaus nichts erklärt, sondern nur eine Unbekannte für die andere eingesetzt. Aber angenommen, die Lähmung wäre wirklich eine toxische, warum sehen wir dann eine solche, ob primär oder sekundär, niemals bei tetanisch infizierten Wunden am Rumpf und Extremitäten? „Die Endausbreitungen des Facialis - sagt Jolly (6) - müssen bei Wunden in der Orbitalregion förmlich in das Gift eingetaucht werden, ${ }^{*}$ - warum finden wir dann aber die Facialislähmung nicht immer in solchen Fällen, sondern häufig den (sogenannten B r u n e r schen) (16) Kopftetanus ohne Facialislähmung? „Der ganze Nerv ist toxisch ${ }^{4}$ - sagt Jolly (17) vom Facialis -, warum ist es dann aber noch niemals (z. B. Nicol ai er) gelungen, mit Gewebsstücken aus ihm Impftetanus zu erzeugen? Dies alles sind noch offene Fragen, und die Hypothese, da $\beta$ die Facialislahmung toxischer Natur ist, steht somit noch auf sehr schwachen Füßen.

Es bleibt die vierte und älteste Ansicht in der Frage zu besprechen, diejenige, die Rose (18) selbst zuerst aussprach und noch heut aufrecht halt. Er sieht die Facialislahmung als eine neuritische an und erkldrt ihre Entstehung auf folgende Weise: Das Trauma erzeugt an der Stelle der Verletzung, die regelmälig im Ausbreitungsgebiete des Facialis gelegen ist, eine entzündliche Schwellıng seiner Nervenscheide, die sich zentripetal fortsetzt. Diese aszendierende Neuritis verläuft symptomlos, wo sie extrakraniell bleibt; erstreckt sie sich aber am Nerven entlang bis in den engen und unnachgiebigen Canalis Fallopiae, so erzeugt dort der Druck des ihn umgebenden Exsudates eine Kompressionslähmung des Nervenstammes. Zwar hat sich auch für diese Theorie leider noch in keinem Falle der pathologisch-anatomische Beweis erbringen lassen, doch ist sie ohne Zweifel einleuchtend und plausibel. Jolly (6) wendet dagegen hauptsächlich folgendes ein: Bei unkomplizierten Kopf-, resp. Gesichtswunden würden unzählige Male kleine und kleinste Aestchen des Facialis lädiert, aber niemals führe das zu einer neuritischen Lahmung des ganzen Stammes - warum solle letztere sich gerade nur bei tetanisch infizierten Wunden entwickeln? Hierauf ist zunächst im Sinne Roses zu erwidern, daß auch beim Kopftetanus die Lähnung keineswegs in allen Fällen eintritt, sondern nur in denjenigen, wo der entzündliche Prozeß bis in den Canalis Fallopiae gewandert ist, und dab sich eben dadurch die Inkonstanz der Facialislähmung beim Kopftetanus, die bei der toxischen Theorie unverständlich bleibt, in zwanglosester Weise erk]art. Aber es darf auch noch auf ein anderes Krankheitsbild hingewiesen werden, bei dem wir in ähnlicher Weise das beobachten, was Jolly anzweifelt: eine Lahmung des ganzen Facialisstammes als Effekt einer aufsteigenden Entzïndung seiner peripherischsten $Z_{w}$ eige: Es $_{>}$ist dic merkwiirdige Kombination von
Zoster faciei mit kompletter Facialislähmung, die, wenngleich selten, doch zu oft, um nur zufallig sein zu können, beobachtet wird. Wie ist hier der innere Zusammenhang der Symptome? Der Herpes zoster des Gesichts beruht auf einer entzïndlichen Affektion des Trigeminus, vorzugsweise in seinem ersten Aste; von diesem greift der Entzündungsproze $\beta$ auf benachbarte oder anastomosierende Endverzweigungen des Facialis iiber und erzeugt, bis zu seinem Stamme hinaufk riechend, eine Lähmung seiner sämtlichen Aeste. Dies ist die Erklärung, die hier übereinstimmend fast alle Beobachter, Strlibing (19), Hess (20), Gellé (21). Tryde (22). Greenough (23), Vernbuil (24) und Létulle (25) geben. Eine Neuritis ascendens als Ursache der Facialislähmung wäre also beim Kopftetanus klinisch nicht ohne Analogon, wenn auch ibr anatomischer Nachweis hier wie dort noch nicht gelungen ist. Immerhin hat diese Annahme hohe Wahrscheinlichkeit für sich, und auch der bisher negative histologische Befund kann dagegen nicht schwer ins Gewicht fallen: denn selbst bei der rheumatischen Facialislähmung, die doch gewiß auf einer exsudativen Entzündung des Nerven beruht, findet sich eine solche, wo es zur Sektion kommt, nicht immer vor, und es muß wohl hier mit der Möglichkeit sub- oder postmortaler Gewebsveränderungen gerechnet werden.

Während eine Facialislähmung ein selbst für den Laien recht augenfälliges und für den Patienten sehr fühlbares Symptom darstellt, gilt dies beides nicht von den Augenmuskellähmungen, und sie können leicht der Beobachtung sowohl des Erkrankten wie seines Arztes entgehen. In unserem Falle bestand, wie oben geschildert wurde, eine komplette, rechtseitige Oculomotorius-, Abducens- und vielleicht auch Trochlearislähmung. Das Vorkommen solcher Lälmmungen bei Kopftetanus wird von einigen Autoren, z. B. von Remak (26) für "dubiös“ erklärt, von anderen, wie Rose, (27) werden sie für terminale Erschöpfungssymptome, also Pseudoparalysen gehalten. Demgegeniiber mub darauf aufmerksam gemacht werden, daß in der ausgezeichneten Kasuistik von Hugo Neumann (28) Augenmuskellähmungen in 14 von 182 Kopftetanusfällen unzweifelliaft nachgewiesen sind und dab dieselben auch in unserem Falle mit Sicherheit - und zwar nicht sub finem vitae, sondern auf der Höhe der Erkrankung - festgestellt worden sind, nachdem sie hinter dem deckenden Verbande wahrscheinlich schon länger bestanden hatten. Was ihren Sitz nnd Ursprung betrifft, so spricht in unserem Falle schon ihre Totalität dafür, daf sie peripherischer Natur sind. Als ihre Entstehungsursache kann wohl nur der Fall auf die Orbita angesehen werden. Denn daß durch ein direkt auf die Orbitaloder Temporalgegend wirkendes Trauma Augenmuskellähmungen hervorgerufen werden können, ist eine durch Erfahrung und Wissenschaft bestätigte Tatsache, die erst jüngst wieder durch einen Fall von Abelsd orf (29) erhärtet worden ist, dessen Patient beim Abspringen von der Trambahn auf die linke Schläfenseite fiel und tags darauf eine totale, linkseitige Oculomotoriuslähmung mit reaktionsloser Pupille aufwies. Wie diese Beobachtung, so mub auch die unsrige dahin erklärt werden, daß die schwere Kontusion der Orbita Hämorrhagien in die Augenmuskelnerven und damit die Lähmungen verursacht hat. In einen kausalen Zusammenhang mit dem Tetanus brauchen somit die Augenmuskellähmungen - zum mindesten in unserem Falle - überhaupt nicht gebracht zu werden. Demnächst wird mit einigen Worten auf den Speichelflub einzugehen sein, unter dem unser Patient einige Tage sehr zu leiden hatte. Rose (30) führt diese Erscheinung beim Kopftetanus auf die zugleich vorhandenen Schlingkrämpfe zurück und meint, es sei nur die Unfähigkeit, den in normaler Menge sezernierten Speichel herunterzuschlucken, die eine Salivation vortäusche. Dieselbe Erklärung gibt Erb (31) für den Speichelflu@ des Bulbärparalytikers, und ähnlich hält man auch beim hydrophobischen Hunde (32) das Geifern für abhängig von seiner bestehendel Schlinglähmung. Allein man hatte in unserem Falle doch den bestimmten Eindruck, dab nicht nur oine scheinbare, auf Schluckbehinderung beruhende Salivation vorlag, sondern dab es ein wirkliches Plus an Speichel war, das stundenwoise unablüssig aus dem Munde des Kranken lief und alle vorgehaltenen Tücher rasch durchnäßte. Verschiedene Beobachter, die gleiches beim Kopftetanus sahen, z. B. Arnsperger, (33) suchen das Symptom nit der begleitenden Facialislähmung in Zusammenhang zu bringen: Der Facialis kommt dabei soweit gewiß in Betracht, als der Speichel in- 
folge des mangelhafterı Lippenschlusses nur auf der gelähmten Seite ausfließt, aber die Hypersekretion selbst wird vom $\mathrm{Fa}$ cialis aus nicht erklärt werden können; denn es ist in hohem Grade zweifelhaft, ob die Störung der Absonderung nur eine einseitige ist, und wenn dies selbst der Fall wäre, so müßte bei Lähmung der in der Chorda tympani verlaufenden Speichelfasern des Facialis gleichseitige Verminderung und nicht Vermehrung der Speichelmenge eintreten. Es liegt wohl näher, an eine reflektorische Reizung des Speichelzentrums in der Medulla, ausgelöst durch die schmerzhaften Kinnbackenkrämpfe, zu denken. Wir sehen Speichelfluß beim Tic douloureux und beim Zahnschmerz nicht selten auf dem Wege des Reflexes auftreten, und ebenso dürfte er beim Trismus durch Irritation sensibler Trigeminusfasern der Mundhöhle zustande kommen. Es kann dieser Auffassung zur Stütze dienen, daß in unserem Falle das Aufhören der Salivation zeitlich zusammenfiel mit dem Aufhören des Trismus, bzw. dem Einsetzen der terminalen Kieferlähmung.

Nachdem im vorstehenden die markantesten Züge unseres Krankheitsbildes durch einige erläuternde Bemerkungen hervorgehoben worden sind, erübrigt es, nocl eines charakteristischen Symptomes Erwähnung zu tun, das merkwürdigerweise nicht vorhanden war: es ist die Nackenstarre, ein typischer, ja pathognomonischer Bestandteil des Wundstarrkrampfes, die vom Anfang bis zum Ende der Erkrankung vollständig fehlte. „lch habe lloch keinen Fall - so schreibt Rose (31), der gründlichste Kenner des Tetanus - von heftigem Wundstarrlkrampf gesehen, wo sich nicht schon in dem elsten Stadium der Kieferstarre eine leichte Beteiligung des Nackens hätte nachweisen lassen." Hier ist dieser Fall. Weder im ersten Stadium, noch in einem späteren hat er auch nur die leiseste Andeutung von Nackenstarre gezeigt. Aber nicht nur in der Nackenmuskulatur fehlte Tonus und Clonus, sondern auch Rücken-, Bauch- und Extremitätenmuskeln, auf die sonst der Tetanus im späteren Verlaufe vom Kopfe aus übergreift, blieben dauernd frei. Im pathologisch-anatomischen Sinne ist dies von ganz besonderer Bedeutung: wenn der traumatische Tetanus auf toxischen Veränderungen in der grauen Substanz des verlängerten Markes beruht, und dieser Prozeb, den die enorme Steigerung der Reflexerregbarkeit anzeigt, sich in der Regel in absteigender Richtung auf das Cervicalmark fortpflanzt, so hat er in unserm Falle an der unteren Grenze der Medulla oblongata Halt gemacht und das Halsmark nicht erreicht. So sind die klinischen Erscheinungen streng auf den Bereich des Kopfes lokalisiert und endgültig beschränkt geblieben, und wenn irgendwo der Name „Kopf"-Tetanus treffend und bezeichnend ist, so ist er es in dem vorliegenden, prägnanten Falle. Auffallend bleibt freilich dabei der Kontrast zwischen der geringen Extensität und der hohen Intensität der Erkrankung, die ein nur so kleines Muskelgebiet umfaßt und doch zu deletärem Ausgange geführt hat - im Widerspruch zu der allgemein geltenden Anschanung, daß, je weniger Muskelgruppen beim Tetanus ergriffen sind, um so gutartiger der Verlauf sich zu gestalten pflegt. Ein anderer, alter Erfahrungssatz, der bis auf Hippokrates zurückgeht, sagt, dab je später ein Tetanus ausbricht und je protrahierter er verläuft, um so wahrscheinlicher die Genesung ist: auch von dieser Regel hat der vorliegende Fall, - ebenso wie ein im vorigen Jahr von J. Friedländer (35) veröffentlichter - leider eine Ausnahme gemacht.

Literatur. 1. Prof Dr. E. Rose, Der Starrkrampf beim Menschen, 1897, S. 338-339. -2 . Ebenda, $S 65-3$. Noth nag e l, Klinische Vorstellung eines Failes von Kopftetanus, Allgemeine Wiener med. Zeitung 1901, No. 10-13. - 4. Rose, Die Kasurstik meines Algemeine Wiener med. Zeitung 1901, No. 10-13. - 4. Rose, Die Kasuıstik meines
Koptetanus, Fall 10,33, 47, 70 u. a. - 5. O1iva, Gazette des hôpitaux 1888, S. 1364. Kopftelanus, Fall 10, $33,47,70$ u. a. -5 . Oliva, Gazette des hôpitaux 1888, S. 1364.
6 . F. Jolly, Ueber einen Fall von Kopftetanas mit Facialislähmung, Vortrag in der -6. F. Jolly, Ueber einen Fall von Kopftetanus mit Facialislähmung, Vortrag in der 7. Goldscheider, Wie wirkt das Tetanusgift auf das Nervensystem?, Zeitschrift für 7. Goldscheider, Wie wirkt das Tetanusgift auf das Nervensystem?, Zeitschrift für
klintsche Medizin l894, Bd 26. - 8. Marie, Recherclies sur la toxine tétanique; Anklintsche Medizin 1894, Bd 26. - 8. Marie, Recherclies sur la toxine tétanique; An-
nales de H'Institut Pasteur 1897, S. 591. - 9 C. Brnnner, Der Kopftetanus beim Tiere nales de l'Institut Pasteur 1897 , S. 591. - 9 C. Brunner, Der Kopftetanus beim Tiere
und die Facialislahinung beim Tetanus hydrophobicus des Menschen, Deutsche Zeitund die Facialislahinung beim Tetanus hydrophobicus des Menschen, Deutsche Zeit-
schrift für Chirurgie 1890 , Bd. $30-10$. Brieger und F raenkel, Untersuchungen schrift fär Chirurgie 1890 , Bd. $30-10$. Brieger und Fraenkel, Untersuchungen
aber Bakteriengifte, Berliner klinische Wochenschrift 1890 , No. 12. - 11. C Brunner, Experimentelle und klinische Studien uber den Kopftetanus, Beiträge zur klinischen Chirurgie, redigiert von P. Bruno, Bd. 9, 10, 12. - 12. H. Oppenhe $1 \mathrm{~m}$, Lehrbuch der Nerventiankheiten 1905 , S. 1424. - 13. Dr. Stieb-Griesheim schreibt auf Anfrage am 6. Dezember 1906: Von dem Trisnus abgesehen, konnte ich sonstige Sym. rage am 6. Dezember

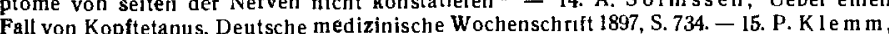
Fall von Kopttetanus, Deutsche medizisitis Ein Fall von Kopftetanus (Tetanus mit Facialislahmung), Deutsche Zeitschrift für Chirurgie 1897 , Bd. 44, S. 44. - 16. C. B runner, Zur Pathogenese des Kopftetanus,
Vortrag von Jolly, Zentralblatt fur Nervenhellkunde tt. Psychiatrie vom 15. Juni 1902. - 18. E. Rose, Der Starrkrampf beint Mensciten S 358. - 19. P. Strü übing, Deutsches Archiv f. klin. Medizin Rd. 37, S 513 - 20 Hess. Virchow-Hirschs Jahrbächer 1859, Bd 2, S 120. - 21. Gellé, Virchow-Hirschs Jahrbiicher 1890, Bd. 2, S. 144 22. Tryde, Schmidts Jahrluücher Bd. 154, S. 273. - 23 Greetiough. Virchow-Hirschs Jahrbicher Bd. 4, S 535. - 24. Verne u11, Sclimidts Jalurbiucher Bd. 162, S. 273. 25. Le tulle. Schmidts Jahrbucher Bd. 194, S. 15 und 26, . 26. E. Reinak, in der ad 17 angeführten Diskussion. - 27. E. Ro se, Der Starrkrampf beim Renak, in der ad 17 angefthirten Diskussion. - 27. E. Ro se, Der Starrkrampt beim Menschen, S. 371 bis 372 . - 28. Hugo Neumann, Sammelreferat íber Kopftetanus, Zentralblatt fut Grenzgebiete der Medizin und Chirurgte 1902, Bd. 5, No. 13. - 29 A belsdorf Demonstration in der Berliner Gesellschaft fur Psychiatrie und Nervenkrankheiten am 5. Marrz 1906 - 30. Rose, Der Starrkrampf beim Menschen, S. 359. - 31. W. Erb Handbuch Jer Krankheiten des Nervensystelns, I, S. 927. - 32. Benedict, Hundswut, Euleuburgs Rcal-Enzyklopadie 1887, Bd. 9, S. 647. - 33. L Arnsperger, Ueber den Roseschen Kopftetanus, Inauguraldissertation, Heldelberg 1901, S. 22. - 34. E. Ro ose, Der Startkrampf beim Menschen, S. $26-35$ Julıus Friedländer, Zur Behand lung des traumatischen Tetanus, Deutsche klınisch-therapeutische Wochenschrift, 1906 , No. 32 